\title{
La régulation des réseaux d'irrigation
}

\author{
par Pierre Rousset
}

Ingénieur à la Société du Canal de Provence

Animateur du groupe de travail «Régulation» de l'AFEID

\section{INTRODUCTION}

Il n'y a pas de conférence ou de manifestation technique de l'AFEID et de la CIID où le thème de la régulation ne soit abordé. Un grand nombre de publications et de thèses y sont consacrées et un groupe de travail de l'AFEID associant bureaux d'études, organismes de recherche et sociétés d'aménagement, en a fait son sujet de réflexion.

Qu'est-ce que la régulation des réseaux d'irrigation pour susciter un tel intérêt et revêtir une telle importance ?

Dans son sens le plus large, la régulation est synonyme de contrôle, ou mieux, de maîtrise du fonctionnement d'un réseau d'irrigation.

Contrairement à l'apparente simplicité des ouvrages, le fonctionnement d'un réseau d'irrigation nécessite un ensemble d'actions concertées, automatiques ou manuelles, qui deviennent de plus en plus complexes avec le niveau de performance recherché

Les performances d'un réseau d'irrigation s'expriment en terme d'efficacité globale (rapport entre le volume d'eau effectivement utilisé par les plantes et le volume d'eau dérivé en amont); elle varie de manière considérable entre moins de $20 \%$ et près de $100 \%$. Cela dépend de nombreux facteurs tels que la nature des canaux ou canalisations, le mode d'irrigation, la nature des sols etc., et pour une large part, de la régulation. Outre l'aspect quantitatif, bien caractérisé par cet indice d'efficacité, l'aspect qualitatif - c'est-àdire sûreté et sécurité de fonctionnement, fourniture de l'eau au bon moment et au bon endroit, etc. - est non moins important et dépend presque exclusivement de la régulation.

Pour compléter cette présentation préliminaire, il est utile de rappeler que la régulation des réseaux d'irrigation met en cuvre de nombreuses techniques et aborde un large éventail de problèmes.

Tout d'abord, il existe deux types d'adductions d'eau aux caractéristiques techniques très différentes:

- les canaux à ciel ouvert qui présentent des temps de réponse très importants,

- les canalisations sous pression qui en revanche ont des réactions quasi instantanées avec des phénomènes transitoires brutaux et souvent dangereux.
Bien entendu, les techniques de régulation et les appareillages à installer sont très différents dans les deux cas.

Ensuite, la régulation doit faire face aux différentes possibilités de fonctionnement :

- fonctionnement normal, soit en régime permanent, soit en régime transitoire,

- fonctionnements anormaux ou dégradés lorsque certains ouvrages sont défaillants et que l'on veut maintenir un service minimum,

- fonctionnement de sécurité en cas d'incident grave mettant en jeu la sécurité des tiers ou des ouvrages.

Enfin, les réseaux constituant le lien entre la ressource et l'utilisateur, leur mode de fonctionnement et donc leur régulation, aura une incidence notable et sera, en retour influencé par la gestion de la ressource et la pratique des irrigations. Il en est de même des contraintes et du coût de l'exploitation-maintenance. Ces problèmes, qui jadis étaient étudiés au niveau de la conception et donnaient lieu ensuite à des règles fixes, ont acquis, ces dernières années, une importance considérable avec l'installation de systèmes centralisés et fortement automatisés. Ils sont maintenant très souvent pris en compte dans les logiciels de supervision et de régulation.

La régulation des réseaux d'irrigation étant une technique aux multiples facettes, une présentation complète nécessiterait un long développement sortant du cadre de cet article. Cet exposé se limitera donc aux grands principes généraux de la régulation des réseaux d'irrigation; il présentera ensuite les récents progrès technologiques et leur impact dans le domaine de l'irrigation. Enfin, il décrira les principaux axes de recherche en France.

\section{LES GRANDS PRINCIPES DE LA RÉGULATION}

D'une manière très générale, les réseaux d'irrigation à surface libre ou sous pression ont une structure arborescente avec un point d'alimentation et de nombreux points de dessertes - ou prises d'irrigation - disséminés le long des adducteurs sur toute la surface équipée. 
En réseau sous pression, les organes de régulation sont limités :

- à l'ouvrage d'alimentation - dérivation, station de pompage, réservoir ou association de ces différents types d'ouvrages,

- aux prises d'irrigation,

- à des ouvrages intermédiaires du type régulateurs de pression pouvant exister en nombre limité mais n'ayant qu'un rôle secondaire dans la régulation d'ensemble.

En réseau à surface libre, en plus de l'ouvrage de tête et des prises d'irrigation, on trouve :

- des ouvrages de répartition des débits à chaque embranchement,

- des régulateurs à intervalles réguliers suivant le type de régulation et la topographie.

Tous ces ouvrages interviennent directement dans le processus de régulation. On peut en déduire immédiatement que la régulation des réseaux à surface libre est plus complexe, du seul fait du nombre d'ouvrages concernés.

De manière très simplificatrice, on peut dire que les différents principes de régulation consistent tous à équilibrer le débit d'alimentation en tête et la somme des débits prélevés aux prises (plus les fuites) en fonction :

- du seul débit d'alimentation en tête ; c'est la commande par l'amont,

- de la somme des débits prélevés; c'est la commande par l'aval,

- du débit d'alimentation, des débits prélevés et des capacités de stockage intermédiaires (dans les biefs de canaux et dans les réservoirs); c'est la commande centralisée.

En réalité, il existe tout un éventail de solutions intermé diaires; là encore nous nous limiterons à évoquer les principales solutions utilisées en les classant par ordre d'apparition chronologique qui correspond très exactement à une complexité croissante.

\subsection{La commande par l'amont}

C'est le principe de régulation le plus ancien, pratiquement le seul à être utilisé jusqu'au début du $x^{e}$ siècle, avant la construction des grands barrages-réservoirs. Le débit d'alimentation est alors fluctuant et incertain, souvent insuffisant en période d'étiage et toute la distribution doit se plier à cette contrainte majeure. Le rôle principal de la régulation est alors d'assurer le partage de l'eau disponible suivant des règles préétablies qui relèvent de la tradition ou de la législation. Il s'agit toujours de réseaux de canaux où les réglages sont effectués manuellement par vannes ou martelières aux prises et aux points de partage à une fréquence relativement faible.

L'irrigation gravitaire requiert un débit minimum de 10 à 30 l/s en tête de parcelle; au dessous d'une certaine surface, le partage du débit n'est donc plus possible et il faut le remplacer par une alimentation discontinue des utilisateurs à tour de rôle, c'est le fameux tour d'eau.

Une certaine automacité peut être obtenue par des seuils, des partiteurs à lame ou des orifices calibrés qui permettent de maintenir dans une large plage de fonctionnement la proportionnalité entre le débit dérivé et le débit du canal d'aljmentation. En France, on trouve ce type de régulation sur la plupart des canaux de Basse Durance, et, à l'étranger, on peut citer l'Inde du Nord Ouest où ce système, qui occupe des surfaces considérables, porte le nom de Warabandi

Avec l'apparition des grands barrages réservoirs, la régulation par l'amont s'est perfectionnée de manière à ajuster le débit d'alimentation amont en fonction des besoins en eau réels. Cela se fait généralement en cumulant d'aval en amont les demandes exprimées par les agriculteurs tous les 10 ou 15 jours.

La fréquence accrue des manœuvres et une plus grande précision des réglages nécessaires à la stricte égalité de l'alimentation et des prélèvements, a entraîné la mise au point d'appareillages plus perfectionnés tels que les vannes à niveau amont constant, les seuils longs dits $«$ bec de canard », les modules à masques. Parallèlement, toute une organisation doit être mise en place pour établir rapidement les tours d'eau et les programmes d'irrigation et ensuite les appliquer.

\subsection{La commande par l'aval}

La difficulté de coordonner et d'appliquer les programmes d'irrigation sur de vastes surfaces a amené les ingénieurs à rechercher l'automaticité totale des canaux et à concevoir la commande par l'aval qui met en œuvre des vannes à niveau aval constant et des canaux à berges horizontales. En théorie, la méthode est applicable à l'ensemble des canaux d'un réseau, mais pour des raisons économiques, elle n'est réellement utilisée que sur les canaux principaux, ce qui a tout de même l'avantage de partager un grand périmètre en unités indépendantes plus réduites et plus faciles à gérer en régulation par l'amont.

La commande par l'aval généralisée a été utilisée plus tardivement (fin des années 50) sur réseaux de conduites. On a alors à faire aux réseaux fonctionnant en libre service à la demande qui se sont depuis, généralisés en France.

Bien évidemment, les conduites sous pression se prêtent plus facilement à ce contrôle par l'aval qui est leur mode de fonctionnement «naturel » à la différence des canaux à surface libre. Cependant l'aptitude des conduites n'est pas suffisante et un certain nombre de mesures doivent être prises pour assurer le bon fonctionnement d'ensemble :

- surdimensionnement des conduites qui doivent faire face aux fluctuations de la demande, (cf. formule de la demande, R. Clément),

- limitation impérative du débit des prises pour assurer une distribution équitable quels que soient l'altitude et l'emplacement des prises,

- automaticité totale de l'alimentation - le plus souvent station de pompage associée à un réservoir - pour satisfaire à tout moment les fluctuations de la demande. Le schéma d'alimentation est parfois beaucoup plus complexe mais le principe demeure.

\subsection{La commande centralisée automatique}

L'association de réseaux de distribution fonctionnant à la demande et de grands adducteurs à surface libre aux réactions très lentes a conduit les ingénieurs, à la fin des années 60, en France (Canal de Provence) et aux Etats Unis (Californian Aqueduct) à concevoir ce nouveau type de régulation rendu possible par les progrès réalisés en matière de télétransmission et d'ordinateur.

Rappelons brièvement que la régulation dynamique du Canal de Provence assure la commande simultanée et coordonnée de tous les régulateurs le long d'un canal et met en œuvre une logique de régulation en deux temps:

- prévision de la demande et réglage prévisionnel d'ensemble.

- correction permanente des réglages pour tenir compte de l'évolution réelle de la demande.

D'autre part, elle nécessite un réseau automatique de télémesure et de télécommande et un ordinateur central.

Cette méthode, appliquée tout d'abord aux canaux principaux, a été progressivement étendue à tous les ouvrages importants en particulier stations de pompage et réservoirs car elle permet:

- d'optimiser le fonctionnement et de réduire le coût des consommations électriques et, de manière plus générale, toutes les dépenses d'exploitation-maintenance,

- d'améliorer la qualité du service grâce à une surveillance continue et des interventions rapides en cas d'incident. 


\section{LES PROGRÈS RÉCENTS DE LA RÉGULATION}

Au cours de la décennie passée, la régulation des réseaux d'irrigation a bénéficié directement des progrès réalisés en matière de télétransmission et de traitement de l'information. Cela concerne :

- les appareils de mesure: des microprocesseurs intégrés traitent les problèmes de dérive et de conversion des mesures augmentant ainsi la précision des résultats et facilitant l'installation des appareils. Les capteurs à sortie numérique existent dès à présent et seront utilisés dans un avenir proche,

- les équipements de télétransmissions assurent maintenant de nombreuses fonctions autres que le codage et l'envoi des informations comme l'enregistrement local des mesures, l'envoi des informations à des destinataires différents en fonction de critères préétablis, et, surtout des fonctions d'automatismes de plus en plus perfectionnées,

- les supports de télétransmission : apparition de la fibre optique, multiplication des réseaux hertziens et, il faut le noter, utilisation du réseau téléphonique commuté dont la fiabilité, la disponibilité et les performances ont fait des progrès considérables.

- les moyens de traitement de l'information ; c'est un lieu commun de rappeler les progrès réalisés sur les matériels et les logiciels. Pratiquement, les ordinateurs centraux peuvent traiter la plupart des problèmes rencontrés en temps réel et localement, les automates programmables assurent des fonctions de contrôle de plus en plus complexes. De plus, la banalisation des ordinateurs personnels a fortement contribue à lever les réticences passées à l'égard des systèmes centrali sés.

Ces progrès techniques se sont accompagnés d'une diminution des prix qui rend l'utilisation de ces moyens beaucoup plus accessible et permet d'envisager des commandes centralisées même dans les pays en voie de développement.

Ce bref tour d'horizon serait incomplet si l'on ne citait la diffusion et la généralisation de modèles de simulation performants et conviviaux en hydraulique sous pression et à surface libre. Ces modèles sont maintenant couramment utilisés pour l'étude du fonctionnement des réseaux et la conception des systèmes de régulation.

Ces progrès concernent toutes les méthodes les plus sophistiquées de régulation et ne touchent qu'indirectement les méthodes plus traditionnelles qui utilisent toujours des appareillages de conception ancienne, tels que modules à masques, vannes à flotteur qui ont été mis au point dans les années 40 et 50 , et même, dans une certaine mesure, les bornes d'irrigation, qui occasionnent toujours d'importantes pertes de charge.

Un effort de recherche dans ce domaine serait sans doute bénéfique.

\section{LES PRINCIPAUX AXES DE RE- CHERCHE EN FRANCE}

En dehors des technologies relatives aux télétransmissions et au traitement de l'information qui ne sont pas spécifiques à l'irrigation, on peut noter deux domaines de recherche en cours.

\subsection{Les automatismes}

Depuis quelques années, les techniques les plus récentes de l'automatisme ont fait une entrée remarquée dans le domaine de la régulation des réseaux d'irrigation. Dans le passé, cette contribution était plus limitée et moins systématique. Cela concerne essentiellement l'hydraulique à surface libre, qui, du fait des retards de propagation et des cascades de régulateurs, met en jeu des systemes physiques complexes difficiles à automatiser et nécessite des études préliminaires importantes.

Plusieurs sociétés d'aménagement, organismes de recherche et bureaux d'étude collaborent dans ce domaine au sein du groupe de travail « Régulation » de l'AFEID.

Nous citerons :

- la Commande Optimale, recherche menée par le CEMAGREF, qui consiste à optimiser simultanément l'ensemble des paramètres de réglage en optimisant un critère défini au préalable qui peut être une fonction coût, performance, sécurité ou autre,

- le Régulateur Proportionnel Intégral Retard, recherche menée par la Société du Canal de Provence, qui consiste à remplacer le très classique régulateur PID (Proportionnel Integral Dérivé) mal adapté aux systèmes à retards importants, par un autre type de régulateur tenant compte des temps de propagation

- la Commande Prédictive, recherche menée par la Compagnie Nationale du Rhône, qui consiste à utiliser un régulateur à modèle interne. Ce modèle, recalé à chaque pas de calcul, permet de déterminer les actions de réglage en fonction d'un certain nombre de contraintes liées à la gestion des ouvrages,

- la Logique Floue, recherche menée par la Compagnie Nationale du Bas Rhône-Lanouedoc, qui consiste à utiliser les acquis de l'intelligence artificielle dans le domaine de la régulation.

\subsection{L'intégration des systèmes de supervision et de la gestion}

Les progrès rapides des télétransmissions et du traitement de l'information se sont faits de manière dispersée et pas du tout standardisée. L'effort de centralisation de la régulation des aménagements hydrauliques et d'intégration des techniques de gestion informatisée se heurte à une difficulté pratique de cohérence des données en temps réel ou en temps différé et de possibilité d'échanges d'informations entre les différents logiciels de traitement. Ce sujet est actuellement étudié dans un programme de recherche européen - le projet WaterCIME - auquel participe la Société du Canal de Provence; son but est de définir une méthodologie générale pour aborder ce problème et de mettre au point et d'expérimenter des solutions pratiques, ouvertes et évolutives.

\section{$\mathrm{V}$ CONCLUSION}

La régulation des réseaux d'irrigation constitue une activité très ancienne mettant en jeu, de manière implicite ou explicite, des méthodes générales actuellement bien définies.

Ces méthodes font appel à des techniques de base multiples comme l'hydraulique et l'automatisme mais reposent sur une organisation préalable de la distribution de l'eau qui doit tenir compte des contraintes naturelles et humaines.

L'évolution de la régulation s'est toujours faite dans le sens d'une liberté accrue mais non illimitée des agriculteurs et d'une amélioration des conditions d'exploitation pour accroître la qualité du service et diminuer les coûts récurrents.

Les techniques modernes de télétransmission et de traitement de l'information sont largement utilisées et ont amené des progrès notables; elles n'ont cependant pas fait disparaître les techniques plus anciennes et traditionnelles qui resteront toujours utilisées dans les décennies à venir. 
La recherche en France dans le domaine de la régulation est importante et permet d'espérer des résultats positifs dans les techniques de l'automatisme et du traitement de l'information.

Pour modérer l'optimisme de ce bilan, on peut cependant regretter l'absence de recherche systématique pour améliorer les appareillages hydro-mécaniques peu sophistiqués qui conditionnent toujours l'efficacité des réseaux d'irrigation dans les pays en voie de développement.

\section{BIBLIOGRAPHIE SOMMAIRE}

\section{Thèses}

11] BURT C.M. - Regulation of sloping canals by automatic downstream control. Utah, 1983.

12] DE LEON M.B. - Contribution à l'amélioration des périmètres irrigués. Montpellier, 1986.

[3] Khaladi A. - Gestion automatique des transferts d'eau en réseau hydraulique maillé à surface libre. Lyon, 1992.

[4] Chevreau G. - Contribution à l'étude de la régulation dans les systèmes hydrauliques à surface libre. Grenoble, 1992.

[5] Deltour J.L. - Application de l'automatisme numérique à la régulation des canaux. Grenoble, 1992.
[6] Malaterre P.O, - Modélisation, analyse et commande optimale LQR d'un canal d'irrigation. Montpellier, 1993.

17] Kosuth P. - Techniques de régulation automatique des systèmes complexes. Application aux systèmes hydrauliques à surface libre. Montpellier, 1994

\section{Manuels - Livres - Brochures}

[8] Goussard J. (ICID). - Automation of irrigation canal.

19] Plusquellec H., Burt C., Walter H.W. (WORLD BANK). - Modern Water Control in Irrigation.

[10] ANKUM Ir P. (IHE). - Flow control in irrigation canal.

[11] Canal System Automation Manual (USBR).

[12] Alsthom Fluide: Brochure technique. AVIO, AVIS AMLL. - Modules à Masques.

[13] Simulation of Irrigation Canal (CEMAGREF)

[14] Projet WaterCIME. - Methodology Guide (EU).

\section{Communications}

Les communications présentées aux symposiums et conférences organisés par l'AFEID, l'ASCE, la Houille Blanche, la Société du Canal de Provence, le CEMAGREF, sur l'irrigation et la régulation. 